\title{
A QUASI-LINEAR PARABOLIC SYSTEM OF CHEMOTAXIS
}

\author{
TAKASI SENBA AND TAKASI SUZUKI
}

Received 15 December 2004; Accepted 21 January 2005

We consider a quasi-linear parabolic system with respect to unknown functions $u$ and $v$ on a bounded domain of $n$-dimensional Euclidean space. We assume that the diffusion coefficient of $u$ is a positive smooth function $A(u)$, and that the diffusion coefficient of $v$ is a positive constant. If $A(u)$ is a positive constant, the system is referred to as socalled Keller-Segel system. In the case where the domain is a bounded domain of twodimensional Euclidean space, it is shown that some solutions to Keller-Segel system blow up in finite time. In three and more dimensional cases, it is shown that solutions to socalled Nagai system blow up in finite time. Nagai system is introduced by Nagai. The diffusion coefficients of Nagai system are positive constants. In this paper, we describe that solutions to the quasi-linear parabolic system exist globally in time, if the positive function $A(u)$ rapidly increases with respect to $u$.

Copyright (c) 2006 T. Senba and T. Suzuki. This is an open access article distributed under the Creative Commons Attribution License, which permits unrestricted use, distribution, and reproduction in any medium, provided the original work is properly cited.

\section{Introduction}

Our purpose is to show the well-posedness of a system of parabolic equations proposed in mathematical biology. Its origin is in Keller and Segel [9], describing the chemotactic aggregation of cellular slime molds which move preferentially toward relatively high concentrations of a chemical secreted by the amoebae themselves. Here, we study the simplified system,

$$
\begin{gathered}
u_{t}=\nabla \cdot(A(u) \nabla u-\chi u \nabla v) \quad \text { in } \Omega \times(0, \infty), \\
\tau v_{t}=d \Delta v-a v+b u \quad \text { in } \Omega \times(0, \infty), \\
\frac{\partial u}{\partial v}=\frac{\partial v}{\partial v}=0 \quad \text { on } \partial \Omega \times(0, \infty), \\
u(\cdot, 0)=u_{0}, \quad v(\cdot, 0)=v_{0} \quad \text { in } \Omega
\end{gathered}
$$

Hindawi Publishing Corporation

Abstract and Applied Analysis

Volume 2006, Article ID 23061, Pages 1-21

DOI 10.1155/AAA/2006/23061 
where $\Omega \subset \mathbf{R}^{N}(N=1,2,3, \ldots)$ is a bounded domain with smooth boundary $\partial \Omega . u_{0}$ and $v_{0}$ are smooth non-negative functions on $\bar{\Omega}$ satisfying

$$
u_{0} \not \equiv 0 \quad \text { in } \Omega, \quad \frac{\partial u_{0}}{\partial \nu}=\frac{\partial v_{0}}{\partial \nu}=\frac{\partial \Delta v_{0}}{\partial \nu}=0 \quad \text { on } \partial \Omega
$$

$\tau, \chi, d, a$ and $b$ are positive constants, and $A(\cdot)$ is a smooth function defined on $[0, \infty)$.

If $A(\cdot)$ is a positive constant, this system describes the mean field of many self-gravitating particles, and the cases $\tau>0$ and $\tau=0$ are referred to as Keller-Segel system and Nagai system, respectively. In this case, if $N=2$, there is a threshold of the initial value in $L^{1}$ norm for existence of the solution globally in time, denoted by $c_{*}$, so that if $\left\|u_{0}\right\|_{1}<\mathcal{c}_{*}$, then the solution exists globally in time and is bounded $([2,5,11,14])$, while blowup of the solution occurs in finite time in the case of $\left\|u_{0}\right\|_{1}>\mathcal{c}_{*}([8,11,12,17])$. This phenomenon was conjectured by [4] and gave a motivation of our previous works on the mass quantization of collapses in the blowup solution. See $[19,20]$ for more details. On the other hand, in $[13,16]$, Nagai and the first author studied

$$
\begin{gathered}
u_{t}=\nabla \cdot(\nabla u-\chi u \nabla \phi(v)) \quad \text { in } \Omega \times(0, \infty), \\
0=d \Delta v-a v+b u \quad \text { in } \Omega \times(0, \infty),
\end{gathered}
$$

and gave necessary and sufficient conditions for the existence of the solution globally in time, assuming that the sensitivity function $\phi=\phi(v)$ takes the form $\phi(v)=v^{p}$ for $p>0$ or $\phi(v)=\log v$. Also, Biler [3] studied the same problem for more general $\phi$. However, system (1.1) has not been studied so much and in this paper, we show that the solution exists globally in time if

$$
\inf _{0 \leq u} A(u)>0, \quad \liminf _{u \rightarrow \infty} \frac{A(u)}{u^{\tilde{\gamma}}}>0
$$

hold for some $\tilde{\gamma}>\max ((N-2) / N, 0)$. Those conditions imply

$$
A(u) \geq \alpha u^{\gamma}+\beta \quad(u \geq 0)
$$

for some $\alpha>0, \beta>0, \gamma \in(\max (0,(N-2) / N), \min (\tilde{\gamma}, 1))$. Henceforth, we put $\tau=d=$ $a=b=1$ for simplicity.

To state the main theorem, we need a few notations. First, given $T \in(0, \infty]$, we set $Q_{T}=\Omega \times(0, T)$ and take the function spaces $H^{\ell}(\bar{\Omega})$ and $H^{\ell, \ell / 2}\left(\overline{Q_{T}}\right)$ as in [10], where $\ell$ is a non-integral positive number. That is, $H^{\ell}(\bar{\Omega})$ denotes the Banach space of continuous functions $\{w(x)\}$ defined on $\bar{\Omega}$, provided with continuous derivatives up to order $[\ell]$ and the finite

$$
|w|_{\Omega}^{(\ell)}=\langle w\rangle_{\Omega}^{(\ell)}+\sum_{j=0}^{[\ell]}\langle w\rangle_{\Omega}^{(j)}=\sum_{|\delta|=[\ell]} \sup _{x^{\prime}, x \in \Omega} \frac{\left|D_{x}^{\delta} w(x)-D_{x}^{\delta} w\left(x^{\prime}\right)\right|}{\left|x-x^{\prime}\right|^{\ell-[\ell]}}+\sum_{j=0}^{[\ell]} \sum_{|\delta|=j} \sup _{x \in \Omega}\left|D_{x}^{\delta} w(x)\right|,
$$

where $\delta=\left(\delta_{1}, \delta_{2}, \ldots, \delta_{N}\right),|\delta|=\delta_{1}+\delta_{2}+\cdots+\delta_{N}$, and $D_{x}^{\delta}=D_{x_{1}}^{\delta_{1}} D_{x_{2}}^{\delta_{2}} \cdots D_{x_{N}}^{\delta_{N}}$. Next, 
$H^{\ell, \ell / 2}\left(\overline{Q_{T}}\right)$ denotes the Banach space of continuous functions $\{w(x, t)\}$ defined on $\overline{Q_{T}}$, provided with continuous derivatives $\left\{D_{t}^{r} D_{x}^{\delta} w(x, t)\right\}$ for $2 r+|\delta|<\ell$ and the finite

$$
\begin{aligned}
|w|_{Q_{T}}^{(\ell)}= & \langle w\rangle_{x, Q_{T}}^{(\ell)}+\langle w\rangle_{t, Q_{T}}^{(\ell / 2)}+\sum_{j=0}^{[\ell]}\langle w\rangle_{Q_{T}}^{(j)} \\
= & \sum_{2 r+|\delta|=[\ell]} \sup _{\left(x^{\prime}, t\right),(x, t) \in Q_{T}} \frac{\left|D_{t}^{r} D_{x}^{\delta} w(x, t)-D_{t}^{r} D_{x}^{\delta} w\left(x^{\prime}, t\right)\right|}{\left|x-x^{\prime}\right|^{\ell-[\ell]}} \\
& +\sum_{2 r+|\delta|=[\ell]} \sup _{\left(x, t^{\prime}\right),(x, t) \in Q_{T}} \frac{\left|D_{t}^{r} D_{x}^{\delta} w(x, t)-D_{t}^{r} D_{x}^{\delta} w\left(x, t^{\prime}\right)\right|}{\left|t-t^{\prime}\right|^{(\ell-[\ell]) / 2}} \\
& +\sum_{j=0}^{[\ell]} \sum_{2 r+|\delta|=j} \sup _{(x, t) \in Q_{T}}\left|D_{t}^{r} D_{x}^{\delta} w(x, t)\right| .
\end{aligned}
$$

Then, the first theorem guarantees the local well-posedness of (1.1).

Theorem 1.1 (time-local solution). If (1.2) holds and

$$
\inf _{u \geq 0} A(u)=\beta>0
$$

in (1.1), then there is $T>0$ such that (1.1) has a unique classical solution $(u, v)$ in $Q_{T}$ satisfying

$$
|u|_{Q_{T}}^{2+\theta}<+\infty, \quad|v|_{Q_{T}}^{2+\theta}<+\infty
$$

for some $\theta \in(0,1)$.

The main theorem is now stated as follows.

Theorem 1.2 (time-global solution). If (1.2) and (1.4) hold in (1.1), then it has a unique classical solution $(u, v)$ in $Q_{\infty}$ satisfying

$$
|u|_{Q_{\infty}}^{2+\theta}<+\infty, \quad|v|_{Q_{\infty}}^{2+\theta}<+\infty
$$

for some $\theta \in(0,1)$.

In Section 2, we consider some linear system related to (1.1). By using some estimates of the solutions and a standard contraction mapping principle, we show Theorem 1.1.

In Section 3, we introduce Lyapunov function of (1.1). By using the Lyapunov function, a maximal regularity in [6] and Moser's technique in [1], we show Theorem 1.2.

\section{Time local solution}

We take $T>0, p>N+2$, and $\ell \in(0,1-(N+2) / p)$. Given

$$
u \in H^{1+\ell, \ell / 2}\left(\overline{Q_{T}}\right) \equiv\left\{w \in H^{\ell, \ell / 2}\left(\overline{Q_{T}}\right) \mid \nabla_{x} u \in H^{\ell, \ell / 2}\left(\overline{Q_{T}}\right)^{N}\right\},
$$


4 A quasi-linear parabolic system of chemotaxis

we apply [10, Theorem IV.5.3] and get the unique solution

$$
(U, v) \in H^{3+\ell,(3+\ell) / 2}\left(\overline{Q_{T}}\right)^{2}
$$

to the system

$$
\begin{gathered}
U_{t}=\nabla \cdot(A(u) \nabla U-U \nabla v) \quad \text { in } Q_{T}, \\
v_{t}=\Delta v-v+u \quad \text { in } Q_{T}, \\
\frac{\partial U}{\partial \nu}=\frac{\partial v}{\partial \nu}=0 \quad \text { on } \partial \Omega \times(0, T), \\
U(\cdot, 0)=u_{0}, \quad v(\cdot, 0)=v_{0} \quad \text { in } \Omega .
\end{gathered}
$$

Thus, we can introduce the operator $\mathscr{F}$ on $\left\{w \in H^{1+\ell, \ell / 2}\left(\overline{Q_{T}}\right) \mid w \geq 0\right.$ in $\left.\overline{Q_{T}}\right\}$ by

$$
\mathscr{F} u=U
$$

Let

$$
\begin{aligned}
& B(M, T) \equiv\left\{u \in H^{1+\ell, \ell / 2}\left(\overline{Q_{T}}\right) \cap C\left([0, T] ; W^{2, p}(\Omega)\right) \mid u \geq 0 \text { in } \overline{Q_{T}},\right. \\
&\left.\partial u / \partial \nu=0 \text { on } \partial \Omega \times[0, T], \max _{0 \leq t \leq T}\left(\|\Delta u\|_{p}^{p}+\|u\|_{p}^{p}\right)^{1 / p} \leq M\right\}
\end{aligned}
$$

for

$$
M=2^{1 / p}\left(\left\|\Delta u_{0}\right\|_{p}^{p}+\left\|u_{0}\right\|_{p}^{p}\right)^{1 / p}
$$

where $\|\cdot\|_{q}$ and $\|\cdot\|_{m, q}$ denote the standard norms in $L^{p}(\Omega)$ and $W^{m, p}(\Omega)$, respectively.

Henceforth, $C_{i}(i=1,2,3,4,5)$ denote positive constants determined by $M$ and $T$, which are monotone increasing in $T$. First, we show the following.

Lemma 2.1. If $u \in B(M, T)$, then the solution $v$ to

$$
v_{t}=\Delta v-v+u \quad \text { in } Q_{T}, \quad \frac{\partial v}{\partial \nu}=0 \quad \text { on } \partial \Omega \times(0, T)
$$

satisfies that

$$
\max _{0 \leq t \leq T}\left(\|\nabla \Delta v(\cdot, t)\|_{p}+\|v(\cdot, t)\|_{p}\right) \leq C_{1} .
$$

Proof. Let $\Delta_{N, q}$ be the Laplacian with homogeneous Neumann boundary condition, realized as an $m$-accretive operator in $L^{q}(\Omega)$, where $q \in(1, \infty)$. Its domain is given by

$$
D\left(\Delta_{N, q}\right) \equiv\left\{w \in W^{2, q}(\Omega) \mid \frac{\partial w}{\partial \nu}=0 \text { on } \partial \Omega\right\}
$$

and henceforth we will write it as $\Delta_{N}$ for simplicity. Then, $-\Delta_{N}+1$ generates the analytic 
semi-group $\left\{e^{t\left(\Delta_{N}-1\right)}\right\}_{t \geq 0}([7])$, and it holds that

$$
v(\cdot, t)=e^{\left(\Delta_{N}-1\right) t} v_{0}+\int_{0}^{t} e^{\left(\Delta_{N}-1\right)(t-s)} u(\cdot, s) d s
$$

On the other hand, we have

$$
\left\|\left(-\Delta_{N}+1\right)^{3 / 2} v_{0}\right\|_{p} \leq C_{2}
$$

from the assumption, and if $u \in B(M, T)$, then it holds that $u(\cdot, t) \in D\left(\Delta_{N}\right)$ and

$$
\left\|\left(-\Delta_{N}+1\right) u(\cdot, t)\right\|_{p} \leq\left(\|\Delta u(\cdot, t)\|_{p}^{p}+\|u(\cdot, t)\|_{p}^{p}\right)^{1 / p} \leq M
$$

for $t \in[0, T]$. Thus, we have from (2.10), (2.11), and (2.12) that

$$
\begin{aligned}
\left\|\left(-\Delta_{N}+1\right)^{3 / 2} v(\cdot, t)\right\|_{p} & \leq C_{2}+\int_{0}^{t}\left\|\left(-\Delta_{N}+1\right)^{1 / 2} e^{\left(\Delta_{N}-1\right)(t-s)}\left(-\Delta_{N}+1\right) u(\cdot, s)\right\|_{p} d s \\
& \leq C_{2}+\int_{0}^{t} \frac{C_{3}}{\sqrt{t-s}} M d s \leq C_{2}+\frac{1}{2} \sqrt{T} C_{3} M,
\end{aligned}
$$

and in particular,

$$
\|\nabla \Delta v(\cdot, t)\|_{p} \leq C_{4}
$$

follows for $t \in[0, T]$. It also holds that

$$
\|v(\cdot, t)\|_{p} \leq C_{5}
$$

and the proof is complete.

Now, we show the following.

Lemma 2.2. There exists $T_{1}>0$ such that

$$
\mathscr{F} B(M, T) \subset B(M, T)
$$

holds for any $T \in\left(0, T_{1}\right]$.

Proof. Henceforth, $C_{i}(i=6,7,8, \ldots, 20)$ denote positive constants depending only on $M$. We have $W^{2, p}(\Omega) \subset W^{1, \infty}(\Omega)$ and it holds that

$$
\sup _{0 \leq t \leq S}\left(\|u(\cdot, t)\|_{1, \infty}+\|v(\cdot, t)\|_{1, \infty}\right) \leq C_{6}
$$

for $u \in B(M, S)$ if $S \in(0,1]$. 
6 A quasi-linear parabolic system of chemotaxis

From the first equation of (2.3), we obtain that

$$
\begin{aligned}
\frac{1}{p} \frac{d}{d t} \int_{\Omega}|U|^{p} d x & =\int_{\Omega} \nabla \cdot(A(u) \nabla U-U \nabla v)|U|^{p-2} U d x \\
& =-(p-1) \int_{\Omega} A(u)|U|^{p-2}|\nabla U|^{2} d x+(p-1) \int_{\Omega}|U|^{p-2} U \nabla v \cdot \nabla U d x \\
& \leq-\beta(p-1) \int_{\Omega}|U|^{p-2}|\nabla U|^{2} d x+(p-1) \int_{\Omega}|U|^{p-1}|\nabla v||\nabla U| d x \\
& \leq-\frac{1}{2} \beta(p-1) \int_{\Omega}|U|^{p-2}|\nabla U|^{2} d x+C_{7} \int_{\Omega}|\nabla v|^{2}|U|^{p} d x .
\end{aligned}
$$

We have $W^{2, p}(\Omega) \subset H^{1+\ell}(\bar{\Omega})$, and hence Lemma 2.1 implies the boundedness of $\nabla v$. Therefore, it follows that

$$
\frac{d}{d t} \int_{\Omega}|U|^{p} d x \leq C_{8} \int_{\Omega}|U|^{p} d x
$$

The first equation of (2.3) implies also that

$$
\begin{aligned}
\frac{1}{p(p-1)} \frac{d}{d t} \int_{\Omega}|\Delta U|^{p} d x & =\frac{1}{p-1} \int_{\Omega}|\Delta U|^{p-2} \Delta U \cdot \Delta U_{t} d x \\
& =-\frac{1}{p-1} \int_{\Omega} \nabla U_{t} \cdot \nabla\left(|\Delta U|^{p-2} \Delta U\right) d x \\
& =-\int_{\Omega} \nabla U_{t} \cdot\left(|\Delta U|^{p-2} \Delta \nabla U\right) d x \\
& =-\int_{\Omega}[\nabla(\nabla \cdot(A(u) \nabla U-U \nabla v)) \cdot \nabla \Delta U]|\Delta U|^{p-2} d x .
\end{aligned}
$$

Here, we have

$$
\begin{aligned}
\nabla\{\nabla \cdot & (A(u) \nabla U-U \nabla v)\} \\
= & A(u) \nabla \Delta U+(\Delta U) \nabla A(u)+\nabla(\nabla A(u) \cdot \nabla U) \\
& -\nabla(\nabla U \cdot \nabla v)-(\Delta v) \nabla U-U \nabla \Delta v \\
= & A(u) \nabla \Delta U+(\Delta U) \nabla A(u)+\sum_{i=1}^{N} \frac{\partial \nabla A(u)}{\partial x_{i}} \frac{\partial U}{\partial x_{i}} \\
& +\sum_{i=1}^{N} \frac{\partial A(u)}{\partial x_{i}} \frac{\partial \nabla U}{\partial x_{i}}-\sum_{i=1}^{N} \frac{\partial \nabla U}{\partial x_{i}} \frac{\partial v}{\partial x_{i}}-\sum_{i=1}^{N} \frac{\partial U}{\partial x_{i}} \frac{\partial \nabla v}{\partial x_{i}} \\
& -(\Delta v) \nabla U-U \nabla \Delta v \\
= & I+I I+I I I+I V-V-V I-V I I-V I I I
\end{aligned}
$$


and those terms are estimated as follows:

$$
\begin{aligned}
& -\int_{\Omega}(I \cdot \nabla \Delta U)|\Delta U|^{p-2} d x \leq-\beta \int_{\Omega}|\nabla \Delta U|^{2}|\Delta U|^{p-2} d x, \\
& -\int_{\Omega}(I I \cdot \nabla \Delta U)|\Delta U|^{p-2} d x \\
& =-\int_{\Omega}(\nabla A(u) \cdot \nabla \Delta U)|\Delta U|^{p-2} \Delta U d x \\
& \leq C_{6}\left\|A^{\prime}\right\|_{L^{\infty}\left(\left[0, C_{6}\right]\right)} \cdot \int_{\Omega}|\nabla \Delta U \| \Delta U|^{p-1} d x \\
& \leq \frac{\beta}{10} \int_{\Omega}|\nabla \Delta U|^{2}|\Delta U|^{p-2} d x+C_{9} \int_{\Omega}|\Delta U|^{p} d x, \\
& -\int_{\Omega}(I I I \cdot \nabla \Delta U)|\Delta U|^{p-2} d x \\
& \leq\left\|A^{\prime}\right\|_{L^{\infty}\left(\left[0, C_{6}\right]\right)} \int_{\Omega} \sum_{i, j=1}^{N}\left|\frac{\partial^{2} u}{\partial x_{i} \partial x_{j}}\right||\nabla U||\nabla \Delta U||\Delta U|^{p-2} d x \\
& +\left\|A^{\prime \prime}\right\|_{L^{\infty}\left(\left[0, C_{6}\right]\right)} \int_{\Omega}|\nabla u|^{2}|\nabla U||\nabla \Delta U||\Delta U|^{p-2} d x \\
& \leq C_{10}\left(\|\Delta u\|_{p}^{p}+\|u\|_{p}^{p}\right)^{1 / p}\|\nabla U\|_{\infty} \\
& \cdot\left\{\int_{\Omega}|\nabla \Delta U|^{2}|\Delta U|^{p-2} d x\right\}^{1 / 2}\|\Delta U\|_{p}^{(p-2) / 2} \\
& +C_{10} C_{6}^{2}\|\nabla U\|_{p}\left\{\int_{\Omega}|\nabla \Delta U|^{2}|\Delta U|^{p-2} d x\right\}^{1 / 2}\|\Delta U\|_{p}^{(p-2) / 2} \\
& \leq \frac{\beta}{10} \int_{\Omega}|\nabla \Delta U|^{2}|\Delta U|^{p-2} d x+C_{11}\left(\|\Delta U\|_{p}^{p}+\|U\|_{p}^{p}\right), \\
& -\int_{\Omega}(I V \cdot \nabla \Delta U)|\Delta U|^{p-2} d x \\
& \leq\left\|A^{\prime}\right\|_{L^{\infty}\left(\left[0, C_{6}\right]\right)} \int_{\Omega}|\nabla u|\left(\sum_{i, j=1}^{N}\left|\frac{\partial^{2} U}{\partial x_{i} \partial x_{j}}\right|\right)|\nabla \Delta U||\Delta U|^{p-2} d x \\
& \leq C_{6}\left\|A^{\prime}\right\|_{L^{\infty}\left(\left[0, C_{6}\right]\right)}\left\{\int_{\Omega}\left(\sum_{i, j=1}^{N}\left|\frac{\partial^{2} U}{\partial x_{i} \partial x_{j}}\right|\right)^{p} d x\right\}^{1 / p} \\
& \cdot\left\{\int_{\Omega}|\nabla \Delta U|^{2}|\Delta U|^{p-2} d x\right\}^{1 / 2}\|\Delta U\|_{p}^{(p-2) / 2} \\
& \leq \frac{\beta}{10} \int_{\Omega}|\nabla \Delta U|^{2}|\Delta U|^{p-2} d x+C_{12}\left(\|\Delta U\|_{p}^{p}+\|U\|_{p}^{p}\right), \\
& -\int_{\Omega}(-V \cdot \nabla \Delta U)|\Delta U|^{p-2} d x \\
& \leq \int_{\Omega}|\nabla v|\left(\sum_{i, j=1}^{N}\left|\frac{\partial^{2} U}{\partial x_{i} \partial x_{j}}\right|\right)|\nabla \Delta U||\Delta U|^{p-2} d x
\end{aligned}
$$


8 A quasi-linear parabolic system of chemotaxis

$$
\begin{aligned}
& \leq C_{6}\left\{\int_{\Omega}\left(\sum_{i, j=1}^{N}\left|\frac{\partial^{2} U}{\partial x_{i} \partial x_{j}}\right|\right)^{p} d x\right\}^{1 / p} \\
& \cdot\left\{\int_{\Omega}|\nabla \Delta U|^{2}|\Delta U|^{p-2} d x\right\}^{1 / 2}\|\Delta U\|_{p}^{(p-2) / 2} \\
& \leq \frac{\beta}{10} \int_{\Omega}|\nabla \Delta U|^{2}|\Delta U|^{p-2} d x+C_{13}\left(\|\Delta U\|_{p}^{p}+\|U\|_{p}^{p}\right), \\
& -\int_{\Omega}(-V I \cdot \nabla \Delta U)|\nabla \Delta U|^{p-2} d x \\
& \leq \int_{\Omega}|\nabla U|\left(\sum_{i, j=1}^{N}\left|\frac{\partial^{2} v}{\partial x_{i} \partial x_{j}}\right|\right)|\nabla \Delta U||\Delta U|^{p-2} d x \\
& \leq C_{14}\|v\|_{2, p}\|\nabla U\|_{\infty}\left\{\int_{\Omega}|\nabla \Delta U|^{2}|\Delta U|^{p-2} d x\right\}^{1 / 2}\|\Delta U\|_{p}^{(p-2) / p} \\
& \leq \frac{\beta}{10} \int_{\Omega}|\nabla \Delta U|^{2}|\Delta U|^{p-2} d x+C_{15}\left(\|\Delta U\|_{p}^{p}+\|U\|_{p}^{p}\right), \\
& -\int_{\Omega}(-V I I \cdot \nabla \Delta U)|\Delta U|^{p-2} d x \\
& \leq \int_{\Omega}|\Delta v||\nabla U||\nabla \Delta U||\Delta U|^{p-2} d x \\
& \leq C_{16}\|v\|_{2, p}\|\nabla U\|_{\infty}\left\{\int_{\Omega}|\nabla \Delta U|^{2}|\Delta U|^{p-2} d x\right\}^{1 / 2}\|\Delta U\|_{p}^{(p-2) / 2} \\
& \leq \frac{\beta}{10} \int_{\Omega}|\nabla \Delta U|^{2}|\Delta U|^{p-2} d x+C_{17}\left(\|\Delta U\|_{p}^{p}+\|U\|_{p}^{p}\right), \\
& -\int_{\Omega}(-V I I I \cdot \nabla \Delta U)|\Delta U|^{p-2} \\
& \leq \int_{\Omega} U|\nabla \Delta v||\nabla \Delta U||\Delta U|^{p-2} d x \\
& \leq\left\{\int_{\Omega}|\nabla \Delta U|^{2}|\Delta U|^{p-2} d x\right\}^{1 / 2}\|\nabla \Delta v\|_{p}\|\Delta U\|_{p}^{(p-2) / 2}\|U\|_{\infty} \\
& \leq \frac{\beta}{10} \int_{\Omega}|\nabla \Delta U|^{2}|\Delta U|^{p-2} d x+C_{18}\left(\|\Delta U\|_{p}^{p}+\|U\|_{p}^{p}\right) .
\end{aligned}
$$

Those inequalities are summarised as

$$
\frac{1}{p(p-1)} \frac{d}{d t} \int_{\Omega}|\Delta U|^{p} d x+\frac{3 \beta}{10} \int_{\Omega}|\nabla \Delta U|^{2}|\Delta U|^{p-2} d x \leq C_{19}\left(\|\Delta U\|_{p}^{p}+\|U\|_{p}^{p}\right),
$$

and therefore, we have

$$
\frac{d}{d t}\left(\|\Delta U\|_{p}^{p}+\|U\|_{p}^{p}\right) \leq C_{20}\left(\|\Delta U\|_{p}^{p}+\|U\|_{p}^{p}\right)
$$


by (2.19). Taking

$$
T_{1}=\min \left(\frac{\log 2}{C_{20}}, S\right)
$$

we have for $T \in\left(0, T_{1}\right]$ that

$$
\sup _{0 \leq t \leq T}\left(\|\Delta U(\cdot, t)\|_{p}^{p}+\|U(\cdot, t)\|_{p}^{p}\right) \leq e^{C_{20} T}\left(\left\|\Delta u_{0}\right\|_{p}^{p}+\left\|u_{0}\right\|_{p}^{p}\right) \leq \frac{1}{2} M^{p} e^{C_{20} T} \leq M^{p},
$$

and the proof is complete.

Next, we show the following.

Lemma 2.3. There is $T_{2} \in\left(0, T_{1}\right]$ satisfying

$$
\max _{0 \leq t \leq T_{2}}\left\|\mathscr{F} u_{1}(\cdot, t)-\mathscr{F} u_{2}(\cdot, t)\right\|_{p}^{p} \leq \frac{1}{2} \max _{0 \leq t \leq T_{2}}\left\|u_{1}(\cdot, t)-u_{2}(\cdot, t)\right\|_{p}^{p}
$$

for any $u_{1}, u_{2} \in B\left(M, T_{2}\right)$.

Proof. We take $T \in\left(0, T_{1}\right]$ and $u_{i} \in B(M, T)$ for $i=1,2$. Let $U_{i}$ and $v_{i}$ be the solutions to (2.3) for $u=u_{i}$. Then, it holds that

$$
v_{2}(\cdot, t)-v_{1}(\cdot, t)=\int_{0}^{t} e^{(s-t)\left(\Delta_{N}-1\right)}\left(u_{1}(\cdot, s)-u_{2}(\cdot, s)\right) d s
$$

and we have

$$
\left\|\nabla\left(v_{2}(\cdot, t)-v_{1}(\cdot, t)\right)\right\|_{p} \leq C_{21} \sqrt{t} \sup _{0 \leq s \leq t}\left\|u_{2}(\cdot, s)-u_{1}(\cdot, s)\right\|_{p} .
$$

Next, we note

$$
\begin{aligned}
\frac{1}{p} \frac{d}{d t} \| & U_{2}(\cdot, t)-U_{1}(\cdot, t) \|_{p}^{p}=\int_{\Omega}\left(U_{2 t}-U_{1 t}\right)\left|U_{2}-U_{1}\right|^{p-2}\left(U_{2}-U_{1}\right) d x \\
= & \int_{\Omega}\left\{\nabla \cdot\left[\left(A\left(u_{2}\right)-A\left(u_{1}\right)\right) \nabla U_{2}\right]+\nabla \cdot\left[A\left(u_{1}\right) \nabla\left(U_{2}-U_{1}\right)\right]\right. \\
& \left.\quad-\nabla \cdot\left[\left(U_{2}-U_{1}\right) \nabla v_{2}\right]-\nabla \cdot\left[U_{1} \nabla\left(v_{2}-v_{1}\right)\right]\right\}\left|U_{2}-U_{1}\right|^{p-2}\left(U_{2}-U_{1}\right) d x \\
= & -(p-1) \int_{\Omega}\left(A\left(u_{2}\right)-A\left(u_{1}\right)\right)\left[\nabla U_{2} \cdot \nabla\left(U_{2}-U_{1}\right)\right]\left|U_{2}-U_{1}\right|^{p-2} d x \\
& \quad-(p-1) \int_{\Omega} A\left(u_{1}\right)\left|\nabla\left(U_{2}-U_{1}\right)\right|^{2}\left|U_{2}-U_{1}\right|^{p-2} d x \\
& +(p-1) \int_{\Omega}\left[\nabla v_{2} \cdot \nabla\left(U_{2}-U_{1}\right)\right]\left|U_{2}-U_{1}\right|^{p-2}\left(U_{2}-U_{1}\right) d x \\
& +(p-1) \int_{\Omega} U_{1}\left[\nabla\left(v_{2}-v_{1}\right) \cdot \nabla\left(U_{2}-U_{1}\right)\right]\left|U_{2}-U_{1}\right|^{p-2} d x \\
= & -I X-X+X I+X I I .
\end{aligned}
$$


Those terms are estimated by Lemmas 2.1 and 2.2 in the following way:

$$
\begin{aligned}
|I X| \leq & (p-1)\left\|A^{\prime}\right\|_{L^{\infty}\left(\left[0, C_{6}\right]\right)}|| u_{2}-u_{1}||_{p}\left\|\nabla U_{2}\right\|_{\infty} \\
& \cdot\left\{\int_{\Omega}\left|\nabla\left(U_{2}-U_{1}\right)\right|^{2}\left|U_{2}-U_{1}\right|^{p-2} d x\right\}^{1 / 2}\left\|U_{2}-U_{1}\right\|_{p}^{(p-2) / 2} \\
\leq & \frac{(p-1) \beta}{4} \int_{\Omega}\left|\nabla\left(U_{2}-U_{1}\right)\right|^{2}\left|U_{2}-U_{1}\right|^{p-2} d x \\
& +C_{22}\left(\| U_{2}-U_{1}||_{p}^{p}+|| u_{2}-u_{1}||_{p}^{p}\right), \\
& -X \leq-\beta(p-1) \int_{\Omega}\left|\nabla\left(U_{2}-U_{1}\right)\right|^{2}\left|U_{2}-U_{1}\right|^{p-2} d x, \\
|X I| \leq & (p-1)|| \nabla v_{2}||_{\infty}\left\{\int_{\Omega}\left|\nabla\left(U_{2}-U_{1}\right)\right|^{2}\left|U_{2}-U_{1}\right|^{p-2} d x\right\}^{1 / 2} \cdot\left\|U_{2}-U_{1}\right\|_{p}^{p / 2} \\
\leq & \frac{\beta(p-1)}{4} \int_{\Omega}\left|\nabla\left(U_{2}-U_{1}\right)\right|^{2}\left|U_{2}-U_{1}\right|^{p-2} d x+C_{23}\left\|U_{2}-U_{1}\right\|_{p}^{p}, \\
|X I I| \leq & (p-1)\left\|U_{1}\right\|_{\infty}\left\{\int_{\Omega}\left|\nabla\left(U_{2}-U_{1}\right)\right|^{2}\left|U_{2}-U_{1}\right|^{p-2} d x\right\}^{1 / 2} \\
& \cdot\left\|U_{2}-U_{1}\right\|_{p}^{(p-2) / 2}\left\|\nabla\left(v_{2}-v_{1}\right)\right\|_{p} \\
\leq & \frac{(p-1) \beta}{4} \int_{\Omega}\left|\nabla\left(U_{2}-U_{1}\right)\right|^{2}\left|U_{2}-U_{1}\right|^{p} d x \\
& +C_{24}\left(\left\|U_{2}-U_{1}||_{p}^{p}+\right\| \nabla\left(v_{2}-v_{1}\right) \|_{p}^{p}\right) .
\end{aligned}
$$

Thus, we obtain

$$
\frac{1}{p} \frac{d}{d t}\left\|U_{2}-U_{1}\right\|_{p}^{p} \leq C_{25}\left(\left\|U_{2}-U_{1}\right\|_{p}^{p}+\left\|u_{2}-u_{1}\right\|_{p}^{p}+\left\|\nabla\left(v_{2}-v_{1}\right)\right\|_{p}^{p}\right)
$$

and hence it follows from (2.30) that

$$
\begin{aligned}
\sup _{0 \leq t \leq T} & \left\|U_{2}(\cdot, t)-U_{1}(\cdot, t)\right\|_{p}^{p} \\
\leq & p C_{25} T\left(\sup _{0 \leq t \leq T}\left\|U_{2}(\cdot, t)-U_{1}(\cdot, t)\right\|_{p}^{p}+\sup _{0 \leq t \leq T}\left\|u_{2}(\cdot, t)-u_{1}(\cdot, t)\right\|_{p}^{p}\right) \\
& +T^{(p / 2)+1} p C_{25} C_{21}^{p} \sup _{0 \leq t \leq T}\left\|u_{2}(\cdot, t)-u_{1}(\cdot, t)\right\|_{p}^{p} .
\end{aligned}
$$

Therefore, for $T_{2}>0$ in

$$
T_{2} \leq T_{1}, \quad p C_{25} T_{2}+T_{2}^{(p / 2)+1} p C_{25} C_{21}^{p} \leq \frac{1}{4}
$$


it holds that

$$
\sup _{0 \leq t \leq T_{2}}\left\|U_{2}(\cdot, t)-U_{1}(\cdot, t)\right\|_{p}^{p} \leq \frac{1}{2} \sup _{0 \leq t \leq T_{2}}\left\|u_{2}(\cdot, t)-u_{1}(\cdot, t)\right\|_{p}^{p}
$$

and the proof is complete.

Now, we can give the following.

Proof of Theorem 1.1. By Lemma 2.2 (with $T=T_{2}$ ) and Lemma 2.3, we have a positive constant $C_{26}$ such that

$$
\begin{aligned}
\left\|U_{t}\right\|_{p} \leq & \|A(u) \Delta U\|_{p}+\left\|A^{\prime}(u) \nabla u \cdot \nabla U\right\|_{p} \\
& +\|\nabla U \cdot \nabla v\|_{p}+\|U \Delta v\|_{p} \leq C_{26}
\end{aligned}
$$

for $u \in B\left(M, T_{2}\right)$. Then [10, Lemma 3.3] guarantees the existence of $\ell \in(0,1)$ such that

$$
|\mathscr{F} u|_{Q_{T_{2}}}^{(\ell)}+|\nabla \mathscr{F} u|_{Q_{T_{2}}}^{(\ell)} \leq C_{27}
$$

for $u \in B\left(M, T_{2}\right)$. Because the set

$$
\mathscr{K} \equiv\left\{\left.u \in B\left(M, T_{2}\right)|| F_{F} u\right|_{Q_{T_{2}}} ^{(\ell)}+|\nabla \mathscr{F} u|_{Q_{T_{2}}}^{(\ell)} \leq C_{27}\right\}
$$

is compact in $X=C\left(\left[0, T_{2}\right] ; L^{p}(\Omega)\right)$, we can apply the standard contraction mapping principle and get a unique fix point of $\mathscr{F}$ on $B=B\left(M, T_{2}\right) \subset X$. This fixed point induces the classical solution $(u, v) \in H^{2+\ell, 1+\ell / 2}\left(\overline{Q_{T_{2}}}\right)^{2}$ to $(1.1)$ on $Q_{T_{2}}$. Conversely, if $(u, v)$ is a classical solution to (1.1) and if $T_{2}>0$ is taken smaller, then, we can regard $(u, v)$ as a fixed point of $\mathscr{F}$ on $B\left(M, T_{2}\right)$. This implies the uniqueness of the classical solution to (1.1) in $Q_{T_{2}}$, and the proof is complete.

\section{Time global solution}

To prove Theorem 1.2, we take

$$
W(u, v)=\int_{\Omega}\left\{B(u)-u v+\frac{1}{2}\left(|\nabla v|^{2}+v^{2}\right)\right\} d x
$$

for

$$
B(u)=\int_{1}^{u} b(s) d s \quad \text { with } b(u)=\int_{1}^{u} \frac{A(s)}{s} d s
$$

and show the following.

Lemma 3.1. If $(u, v)$ is the classical solution to (1.1), then it holds that

$$
\frac{d}{d t} W(u, v)+\int_{\Omega}\left(\left|v_{t}\right|^{2}+u|\nabla(b(u)-v)|^{2}\right) d x=0
$$


12 A quasi-linear parabolic system of chemotaxis

Proof. From the first equation of (1.1) we have

$$
\begin{gathered}
\frac{d}{d t} \int_{\Omega}(B(u)-u v) d x+\int_{\Omega} u v_{t} d x=\int_{\Omega} u_{t}(b(u)-v) d x \\
=-\int_{\Omega} u|\nabla(b(u)-v)|^{2} d x=0,
\end{gathered}
$$

and the second equation implies that

$$
\int_{\Omega} u v_{t} d x=\int_{\Omega}\left(v_{t}-\Delta v+v\right) v_{t} d x=\int_{\Omega}\left|v_{t}\right|^{2} d x+\frac{1}{2} \frac{d}{d t} \int_{\Omega}\left(|\nabla v|^{2}+v^{2}\right) d x
$$

Thus, the conclusion follows from (3.4) and (3.5).

We have

$$
\begin{array}{ll}
b(u) \geq \frac{\alpha}{\gamma}\left(u^{\gamma}-1\right)+\beta \log u & (u \geq 1) \\
b(u) \leq \frac{\alpha}{\gamma}\left(u^{\gamma}-1\right)+\beta \log u & (0<u \leq 1)
\end{array}
$$

for $\alpha, \beta, \gamma$ given in (1.5), and hence it follows that

$$
B(u) \geq B_{0}(u) \equiv \frac{\alpha}{\gamma(1+\gamma)}\left(u^{1+\gamma}-(1+\gamma) u+\gamma\right)+\beta(u \log u-u+1)
$$

for $u \in(0, \infty)$. Therefore, we have $W_{0}(u, v) \leq W(u, v)$ for $W_{0}=W_{0}(u, v)$ defined by

$$
W_{0}(u, v) \equiv \int_{\Omega}\left\{B_{0}(u)-u v+\frac{1}{2}\left(|\nabla v|^{2}+v^{2}\right)\right\} d x
$$

Henceforth, $T_{\max }$ denotes the maximal existence time of the classical solution $(u, v)$ to (1.1), and $C_{i}(i=28,29,30, \ldots, 40)$ are positive constants independent of $T_{\max }$. We have

$$
\|u(\cdot, t)\|_{1}=\left\|u_{0}\right\|_{1}
$$

and the following lemma is a consequence of the estimate on the fundamental solution to the heat equation, described in [21]. The proof is immediate and is omitted.

Lemma 3.2. If $p$ is in $[1, N /(N-2))$ and $[1, \infty)$ for $N \geq 3$ and $N=1,2$, respectively, then it follows that

$$
\sup _{0 \leq t<T_{\max }}\|v(\cdot, t)\|_{p} \leq C_{28}
$$

Now, we show the following.

Lemma 3.3. If $(u, v)$ is the classical solution to (1.1), then it holds that

$$
\begin{gathered}
\sup _{0 \leq t<T_{\max }} W_{0}(u(\cdot, t), v(\cdot, t)) \leq C_{29} \\
\sup _{0 \leq t<T_{\max }}\left(\|u(\cdot, t)\|_{1+\gamma}^{1+\gamma}+\|v(\cdot, t)\|_{1,2}^{2}\right) \leq C_{29} .
\end{gathered}
$$


Proof. First, we have

$$
\sup _{0 \leq t<T_{\max }} W_{0}(u(\cdot, t), v(\cdot, t)) \leq \sup _{0 \leq t<T_{\max }} W(u(\cdot, t), v(\cdot, t)) \leq W\left(u_{0}, v_{0}\right)
$$

with

$$
\int_{\Omega} u v d x \leq\|u\|_{1+\gamma}\|v\|_{(1+\gamma) / \gamma}
$$

Then, Gagliardo-Nirenberg's inequality is indicated as

$$
\|w\|_{q} \leq K_{p, q}\left(\|\nabla w\|_{2}^{2}+\|w\|_{2}^{2}\right)^{\theta / 2}\|w\|_{p}^{1-\theta}
$$

for $w \in H^{1}(\Omega)$, where $p, q \in[1, \infty]$, and $\theta \in(0,1)$ are in

$$
-\frac{N}{q}=\theta\{1-(N / 2)\}-(1-\theta) \frac{N}{p} .
$$

In the case of $N \geq 3$, we take $q=(\gamma+1) / \gamma, \theta=2 \gamma /(\gamma+1)$ and $p=N(1-\gamma) /(2 \gamma)$ to adjust (3.15). We have $p \in[1, N /(N-2))$ from the assumption, and (3.14) assures $C_{\varepsilon}>0$ for any $\varepsilon>0$ satisfying

$$
\varepsilon\|\nabla v\|_{2}^{2} \geq\|v\|_{(\gamma+1) / \gamma}^{(\gamma+1) / \gamma}-C_{\varepsilon}
$$

by Lemma 3.2. The case $N=1,2$ is easier to guarantee (3.16). Therefore, we have from (3.12) that

$$
W\left(u_{0}, v_{0}\right) \geq \int_{\Omega}\left\{\frac{\alpha}{2 \gamma(\gamma+1)} u^{1+\gamma}+\frac{1}{4}\left(|\nabla v|^{2}+v^{2}\right)\right\} d x-C_{30} .
$$

This implies the second inequality, and the proof is complete.

LEMMA 3.4. If $q \geq(3+\gamma) / 2$ and

$$
\sup _{0 \leq t<T_{\max }}\|u(\cdot, t)\|_{q+(\gamma-1) / 2} \leq C_{32}
$$

then it holds that

$$
\sup _{0 \leq t<T_{\max }}\|u(\cdot, t)\|_{2 q-1} \leq C_{33} .
$$

Proof. From the first equation of (1.1) we have

$$
\begin{aligned}
\int_{\Omega} u_{t} u^{2 q-2} d x & =\int_{\Omega} \nabla \cdot(A(u) \nabla u-u \nabla v) u^{2 q-2} d x \\
& \leq-(2 q-2) \int_{\Omega}\left(\alpha u^{\gamma}+\beta\right) u^{2 q-3}|\nabla u|^{2} d x+(2 q-2) \int_{\Omega} u^{2 q-2} \nabla u \cdot \nabla v d x \\
& =-(2 q-2) \int_{\Omega}\left(\alpha u^{\gamma}+\beta\right) u^{2 q-3}|\nabla u|^{2} d x-\frac{2 q-2}{2 q-1} \int_{\Omega} u^{2 q-1} \Delta v d x .
\end{aligned}
$$


14 A quasi-linear parabolic system of chemotaxis

On the other hand, the second equation of (1.1) implies

$$
\left(e^{t /(4 q)} v\right)_{t}=\left(\Delta-1+\frac{1}{4 q}\right)\left(e^{t /(4 q)} v\right)+e^{t /(4 q)} u
$$

and the maximal regularity theorem [6] guarantees

$$
\begin{aligned}
& \int_{0}^{t}\left\|\left(e^{s /(4 q)} v\right)_{s}\right\|_{2 q}^{2 q} d s+\int_{0}^{t}\left\|\left(\Delta-1+\frac{1}{4 q}\right)\left(e^{s /(4 q)} v\right)\right\|_{2 q}^{2 q} d s \\
& \leq C_{34}\left\{\int_{0}^{t}\left\|\left(\Delta-1+\frac{1}{4 q}\right) e^{\{\Delta-1+(1 /(4 q))\} s} v_{0}\right\|_{2 q}^{2 q} d s+\int_{0}^{t}\left\|e^{s /(4 q)} u\right\|_{2 q}^{2 q} d s\right\}
\end{aligned}
$$

with $C_{34}$ independent of $t \geq 0$. This implies

$$
\int_{0}^{t} e^{s / 2}\|\Delta v\|_{2 q}^{2 q} d s \leq C_{35}\left\{t\left\|v_{0}\right\|_{2,2 q}^{2 q}+\int_{0}^{t} e^{s / 2}\|u\|_{2 q}^{2 q} d s\right\}
$$

We can apply (3.14) for $q=r$ and $p=1$ if $\theta=N(1-(1 / r)) /(1+(N / 2)) \in(0,1)$, or equivalently $1<r<2 N /(N-2)$. Putting

$$
k=(2 q+\gamma-1) / 2, \quad r=2 q / k,
$$

we have

$$
r=\frac{4 q}{2 q+\gamma-1} \in\left[2,2+\frac{1-\gamma}{1+\gamma}\right]
$$

and therefore, that inequality is applicable to $w=u^{k}, q=r$ and $p=1$ :

$$
\left\|u^{k}\right\|_{r} \leq K_{r}\left(\left\|\nabla u^{k}\right\|_{2}^{2}+\left\|u^{k}\right\|_{2}^{2}\right)^{\theta / 2}\left\|u^{k}\right\|_{1}^{1-\theta}
$$

Here, we have

$$
K=\max _{2 \leq r \leq 2+(1-\gamma) /(1+\gamma)} K_{r}<+\infty, \quad r \theta=\frac{2 N}{N+2}(r-1)<2,
$$

and any $\varepsilon>0$ admits $C_{\varepsilon}>0$ such that

$$
\begin{aligned}
\left\|u^{k}\right\|_{r}^{r} & \leq K^{r}\left(\left\|\nabla u^{k}\right\|_{2}^{2}+\left\|u^{k}\right\|_{2}^{2}\right)^{r \theta / 2}\left\|u^{k}\right\|_{1}^{r(1-\theta)} \\
& \leq \varepsilon\left(\left\|\nabla u^{k}\right\|_{2}^{2}+\left\|u^{k}\right\|_{2}^{2}\right)+C_{\varepsilon}\left\|u^{k}\right\|_{1}^{r(1-\theta)(2 /(r \theta))^{\prime}},
\end{aligned}
$$


where

$$
\begin{aligned}
\left(\frac{2}{r \theta}\right)^{\prime} & =\frac{2 /(r \theta)}{(2 /(r \theta))-1}=\frac{N+2}{(N+2)-N(r-1)} \\
& \leq \begin{cases}3(1+\gamma) /(1+3 \gamma) & \text { if } N=1 \\
(1+\gamma) / \gamma & \text { if } N=2 \\
(N+2)(N-1) /(N-2) & \text { if } N \geq 3\end{cases} \\
& =\kappa
\end{aligned}
$$

with

$$
\Lambda=\Lambda(q)=r(1-\theta)\left(\frac{2}{r \theta}\right)^{\prime}=\frac{2 N-(N-2) r}{2 N+2-N r}
$$

Thus, we have

$$
\begin{aligned}
C_{35} \int_{0}^{t} e^{s / 2}\|u\|_{2 q}^{2 q} d s \leq & (q-1)(2 q-1) \alpha \int_{0}^{t} \int_{\Omega} e^{s / 2} u^{2 q+\gamma-3}|\nabla u|^{2} d x d s \\
& +C_{36} \int_{0}^{t} e^{s / 2}\left(\|u\|_{q+(\gamma-1) / 2}^{q+(\gamma-1) / 2}+1\right)^{\Lambda} d s .
\end{aligned}
$$

On the other hand, by (3.26), (3.29) and $\left\|u^{k}\right\|_{r}^{r}=\|u\|_{2 q}^{2 q}$, we have

$$
\begin{gathered}
-(q-1)(2 q-1) \int_{\Omega} \alpha u^{2 q+\gamma-3}|\nabla u|^{2} d x+(2 q-2) \int_{\Omega} u^{2 q-1}|\Delta v| d x \\
\leq-\frac{(q-1)(2 q-1)}{k^{2}} \alpha\left\|\nabla u^{k}\right\|_{2}^{2}+(2 q-2)\|u\|_{2 q}^{2 q-1}\|\Delta v\|_{2 q} \\
\leq-\frac{1}{2}\|u\|_{2 q-1}^{2 q-1}+\|\Delta v\|_{2 q}^{2 q}+C_{37}\left(\|u\|_{q+(\gamma-1) / 2}^{q+(\gamma-1) / 2}+1\right)^{\Lambda} .
\end{gathered}
$$

From (3.32) and (3.20) we obtain

$$
\begin{aligned}
& \frac{d}{d t}\|u\|_{2 q-1}^{2 q-1}+(q-1)(2 q-1) \alpha \int_{\Omega} u^{2 q+\gamma-3}|\nabla u|^{2} d x \\
& \quad \leq-(q-1)(2 q-1) \alpha \int_{\Omega} u^{2 q+\gamma-3}|\nabla u|^{2} d x+(2 q-2) \int_{\Omega} u^{2 q-1}|\Delta v| d x \\
& \quad \leq-\frac{1}{2}\|u\|_{2 q-1}^{2 q-1}+\|\Delta v\|_{2 q}^{2 q}+C_{37}\left(\|u\|_{q+(\gamma-1) / 2}^{q+(\gamma-1) / 2}+1\right)^{\Lambda} .
\end{aligned}
$$

Multiplying $e^{t / 2}$ for the above inequality, integrating over $[0, t]$ and using (3.23) and (3.31), we now get that

$$
\begin{gathered}
e^{t / 2}\|u\|_{2 q-1}^{2 q-1}+(q-1)(2 q-1) \alpha \int_{0}^{t} e^{s / 2} \int_{\Omega} u^{2 q+\gamma-3}|\nabla u|^{2} d x d s \\
\leq \int_{0}^{t} e^{s / 2}\|\Delta v\|_{2 q}^{2 q} d s+C_{38} e^{t / 2}\left(L_{q+(\gamma-1) / 2}+1\right)^{\Lambda}
\end{gathered}
$$


or

$$
\|u(\cdot, t)\|_{2 q-1}^{2 q-1} \leq C_{39}\left(L_{q+(\gamma-1) / 2}+1\right)^{\Lambda}
$$

where

$$
L_{q+(\gamma-1) / 2}=\sup _{0 \leq t<T_{\max }}\|u\|_{q+(\gamma-1) / 2}^{q+(\gamma-1) / 2}
$$

Thus, the proof is complete.

Now, we show the uniform boundedness of the solution.

Proposition 3.5. It holds that

$$
\sup _{0 \leq t<T_{\max }}\left(\|u(\cdot, t)\|_{\infty}+\|v(\cdot, t)\|_{\infty}\right) \leq C_{40} .
$$

Proof. By means of Lemmas 3.3 and 3.4, we have

$$
\sup _{0 \leq t<T_{\max }}\|u(\cdot, t)\|_{q}<+\infty \quad \text { for any } q \geq 1+\gamma .
$$

We have $D\left(\left(-\Delta_{N, 2 N+1}+1\right)^{3 / 4}\right) \subset W^{1, \infty}(\Omega)$, and hence it holds that

$$
\begin{aligned}
& \|\nabla v(\cdot, t)\|_{\infty} \leq C_{41}\left\|\left(-\Delta_{N}+1\right)^{3 / 4} v(\cdot, t)\right\|_{2 N+1} \\
& \leq C_{41}\left(\left\|\left(-\Delta_{N}+1\right)^{3 / 4} e^{-t\left(\Delta_{N}-1\right)} v_{0}\right\|_{2 N+1}\right. \\
& \left.\quad \quad+\int_{0}^{t}\left\|\left(-\Delta_{N}+1\right)^{3 / 4} e^{-(t-s)\left(\Delta_{N}-1\right)} u(\cdot, s)\right\|_{2 N+1} d s\right) \\
& \leq C_{42}\left(\left\|\left(-\Delta_{N}+1\right)^{3 / 4} v_{0}\right\|_{2 N+1}+\int_{0}^{t}(t-s)^{-3 / 4} e^{-(t-s)}\|u(\cdot, t)\|_{2 N+1} d s\right) \\
& \leq C_{43}\left(\left\|v_{0}\right\|_{2,2 N+1}+\sup _{0 \leq t<T_{\max }}\|u(\cdot, t)\|_{2 N+1}\right) \leq C_{44} .
\end{aligned}
$$

Taking $q \geq 1+\gamma$ and recalling (3.24), we have

$$
\begin{aligned}
\frac{1}{2 q-1} & \frac{d}{d t} \int_{\Omega} u^{2 q-1} d x+(2 q-2) \int_{\Omega}\left(\alpha u^{\gamma}+\beta\right) u^{2 q-3}|\nabla u|^{2} d x \\
& \leq(2 q-2) \int_{\Omega} u^{2 q-2} \nabla u \cdot \nabla v d x \\
& \leq(2 q-2)\|\nabla v\|_{\infty}\left(\int_{\Omega} u^{2 q+\gamma-3}|\nabla u|^{2} d x\right)^{1 / 2} \cdot\left(\int_{\Omega} u^{2 q-\gamma-1}\right)^{1 / 2} \\
& \leq \alpha(q-1) \int_{\Omega} u^{2 q+\gamma-3}|\nabla u|^{2} d x+\frac{q-1}{\alpha}\|\nabla v\|_{\infty}^{2} \int_{\Omega} u^{2 q-\gamma-1} d x \\
& \leq \alpha(q-1) \int_{\Omega} u^{2 q+\gamma-3}|\nabla u|^{2} d x+\frac{q-1}{\alpha}\|\nabla v\|_{\infty}^{2}\left(\|u\|_{2 q}^{2 q}+|\Omega|\right),
\end{aligned}
$$


or

$$
\begin{gathered}
\frac{d}{d t} \int_{\Omega} u^{2 q-1} d x+\frac{\alpha(q-1)(2 q-1)}{k^{2}} \int_{\Omega}\left|\nabla u^{k}\right|^{2} d x \\
\leq \frac{(q-1)(2 q-1)}{\alpha}\|\nabla v\|_{\infty}^{2}\left(\|u\|_{2 q}^{2 q}+|\Omega|\right) .
\end{gathered}
$$

Here, we have from (3.24), (3.26) and (3.39) that

$$
\begin{aligned}
& \left(\frac{(q-1)(2 q-1)}{\alpha}\|\nabla v\|_{\infty}^{2}+1\right)\left(\|u\|_{2 q}^{2 q}+|\Omega|\right) \\
& \leq C_{44} q^{2}\left\{\int_{\Omega}\left|\nabla u^{k}\right|^{2} d x+\int_{\Omega} u^{2 k} d x\right\}^{r \theta / 2} \cdot\left\{\int_{\Omega} u^{k} d x\right\}^{r(1-\theta)}+C_{44} q^{2} \\
& \leq \frac{\alpha(q-1)(2 q-1)}{k^{2}}\left\{\int_{\Omega}\left|\nabla u^{k}\right|^{2} d x+\int_{\Omega} u^{2 k} d x\right\} \\
& \quad+C_{45} q^{2(2 /(r \theta))^{\prime}}\left\{\int_{\Omega} u^{k} d x\right\}^{r(1-\theta)(2 /(r \theta))^{\prime}}+C_{44} q^{2}
\end{aligned}
$$

In other words,

$$
\begin{aligned}
& \left(\frac{(q-1)(2 q-1)}{\alpha}\|\nabla v\|_{\infty}^{2}+1\right)\left(\|u\|_{2 q}^{2 q}+|\Omega|\right) \\
& \leq \frac{\alpha(q-1)(2 q-1)}{k^{2}}\left\{\int_{\Omega}\left|\nabla u^{k}\right|^{2} d x+\|u\|_{2 q+\gamma-1}^{2 q+\gamma-1}\right\} \\
& \quad+C_{45} q^{2(2 /(r \theta))^{\prime}}\left\{\int_{\Omega} u^{k} d x\right\}^{r(1-\theta)(2 /(r \theta))^{\prime}}+C_{44} q^{2}
\end{aligned}
$$

Here and henceforce, $C_{i}(i=41,42,43, \ldots, 50)$ denote positive constants independent of $q \geq 1+\gamma$ and $T_{\max }$.

Combining this with (3.30) and (3.41), we get that

$$
\frac{d}{d t}\|u\|_{2 q-1}^{2 q-1}+\|u\|_{2 q}^{2 q} \leq C_{46} q^{2 \kappa}\left\{\int_{\Omega} u^{q+((\gamma-1) / 2)} d x+1\right\}^{\Lambda}
$$

Because of $\gamma \in(0,1)$ and (3.29), we have

$$
\left(\sup _{0 \leq t<T_{\max }}\|u(\cdot, t)\|_{2 q-1}^{2 q-1}+1\right) \leq C_{47} q^{2 \kappa}\left(\sup _{0 \leq t<T_{\max }}\|u(\cdot, t)\|_{q}^{q}+1\right)^{\Lambda} .
$$

For $q=2^{j-1}+1$, we put

$$
\alpha_{j}=\sup _{0 \leq t<T_{\max }}\|u(\cdot, t)\|_{2 q-1}^{2 q-1}+1, \quad \Lambda_{j}=\max \left(2, \tilde{\Lambda}_{j}\right),
$$

where $\tilde{\Lambda}_{j}=\Lambda(q)=\Lambda\left(2^{j-1}+1\right)$. 
18 A quasi-linear parabolic system of chemotaxis

Here, we have $\Lambda_{j} \leq 2\left(1+C_{48} 2^{-j}\right)$. In use of $\Lambda_{j} \geq 2$, we apply (3.45) as $q=2^{j-1}+1$ for $j=0,1,2, \ldots$. Then, we get that

$$
\begin{aligned}
\log \alpha_{j} & \leq \log C_{47}+2 \kappa \log \left(2^{j-1}+1\right)+\Lambda_{j} \log \alpha_{j-1} \\
& \leq \log C_{47}+2 \kappa j \log 2+\Lambda_{j} \log \alpha_{j-1}
\end{aligned}
$$

or

$$
\log \alpha_{j}+2 \kappa(j+2) \log 2+\log C_{47} \leq \Lambda_{j}\left(\log \alpha_{j-1}+2 \kappa(j+1) \log 2+\log C_{47}\right),
$$

which implies

$$
\log \alpha_{j}+2 \kappa(j+2) \log 2+\log C_{47} \leq\left(\prod_{i=1}^{j} \Lambda_{i}\right)\left(\log \alpha_{0}+4 \kappa \log 2+\log C_{47}\right)
$$

or

$$
\alpha_{j} \leq\left(C_{47} 2^{4 \kappa} \alpha_{0}\right)^{\prod_{i=1}^{j} \Lambda_{i}}\left(C_{47} 2^{2 \kappa(j+2)}\right)^{-1}
$$

Hence, we obtain

$$
\sup _{0 \leq t<T_{\max }}\|u(\cdot, t)\|_{2^{j}+1} \leq\left(C_{47} 2^{4 \kappa} \alpha_{0}\right)^{\left(\prod_{i=1}^{j} \Lambda_{i}\right) /\left(2^{j}+1\right)}\left(C_{47} 2^{2 \kappa(j+3)}\right)^{-1 /\left(2^{j}+1\right)} .
$$

Here, we apply

$$
\prod_{i=1}^{j} \Lambda_{i} \leq 2^{j} \exp \left(C_{48} \sum_{i=1}^{j} 2^{-i}\right) \leq 2^{j} e^{C_{48}}=2^{j} C_{49}
$$

and get that

$$
\sup _{0 \leq t<T_{\max }}\|u(\cdot, t)\|_{\infty} \leq C_{50}\left(\sup _{0 \leq t<T_{\max }}\|u(\cdot, t)\|_{2}^{2}+1\right)^{C_{49}} .
$$

The desired conclusion follows from this inequality and (3.38) with $q=2$.

Let us confirm that Proposition 3.5 says that $\sup _{0 \leq t<T_{\max }}\|u(\cdot, t)\|_{\infty}$ and $\sup _{0 \leq t<T_{\max }} \| v(\cdot$, $t) \|_{\infty}$ are estimated from above by constants independent of $T_{\max }$. We recall $p>N+2$ and $\ell \in(0,1-(N+2) / p)$. Then, (3.23) with $2 q=p$ means that

$$
\int_{0}^{t} e^{s / 2}\|\Delta v\|_{p}^{p} d s \leq C_{51}\left(t\left\|v_{0}\right\|_{2, p}^{p}+\int_{0}^{t} e^{s / 2}\|u\|_{p}^{p} d s\right)
$$

and hence we obtain

$$
\int_{0}^{t} e^{(s-t) / 2}\|\Delta v\|_{p}^{p} d s \leq C_{52}\left(t e^{-t / 2}\left\|v_{0}\right\|_{2, p}^{p}+\sup _{0 \leq t<T_{\max }}\|u(\cdot, t)\|_{p}^{p}\right) .
$$

Here, $C_{i}(i=51,52)$ are independent of $t \in\left[0, T_{\max }\right)$. 
We take $\tau \in\left(0,(1 / 2) \min \left(T_{\max }, 1\right)\right)$ in $1<T_{\max }-\tau$ without loss of generality. Moreover, we take $T \in\left[\tau, T_{\max }-1\right)$. Henceforce, $C_{i}(i=53,54,55, \ldots, 58)$ denote positive constants independent of $T \in\left[\tau, T_{\max }\right)$ and $T_{\max }$. In use of the reflection with respect to $\partial \Omega$, we have a domain $\widetilde{\Omega} \supset \bar{\Omega}$ with smooth boundary and the extension $(\tilde{u}, \widetilde{v})$ of $(u, v)$ defined on $\widetilde{\Omega}$. Then, we can apply [10, Theorem 3.1] for $(\tilde{u}, \widetilde{v})$, and find $\theta \in(0,1)$ such that

$$
|\tilde{u}|_{\Omega \times(T, T+1)}^{(\theta)} \leq C_{53} .
$$

In fact, $\int_{T}^{T+1}\|\Delta v\|_{p}^{p} d t$ is estimated from above independent of $T$ and $T_{\max }$ by (3.55), and this implies (3.56). Then, it holds that

$$
|u|_{\Omega \times(T, T+1)}^{(\theta)} \leq C_{53},
$$

and the parabolic regularity guarantees that

$$
|u|_{Q_{T_{\max }}^{(\theta)}}^{(\theta)} C_{54}
$$

Then,

$$
|v|_{Q_{T_{\max }}}^{(2+\theta)} \leq C_{55}
$$

follows from [10, Theorem 5.3].

We take $\zeta \in C^{\infty}(\mathbf{R})$ in $0 \leq \zeta \leq 1$ and

$$
\zeta(s)= \begin{cases}1 & \text { if } \tau \leq s \\ 0 & \text { if } s \leq 0\end{cases}
$$

and set $\zeta_{T}(s)=\zeta(s-T)$. The function $W=\mathscr{A}(u)$ defined by

$$
\mathscr{A}(s)=\int_{0}^{s} A\left(s^{\prime}\right) d s^{\prime}
$$

satisfies that

$$
W_{t}=A \Delta W-(\nabla v \cdot \nabla W+A u \Delta v)
$$

and hence we can deduce for $W \zeta_{T}$ that

$$
\begin{gathered}
\left(W \zeta_{T}\right)_{t}=A \Delta\left(W \zeta_{T}\right)-\left(\nabla v \cdot \nabla\left(W \zeta_{T}\right)\right) \\
+\left(W \zeta_{T}^{\prime}-A u \Delta v \zeta_{T}\right) \quad \text { in } \Omega \times[T, T+1], \\
\frac{\partial}{\partial \nu}\left(W \zeta_{T}\right)=0 \quad \text { on } \partial \Omega \times[T, T+1], \\
W(\cdot, T) \zeta_{T}(T)=0 \quad \text { in } \Omega .
\end{gathered}
$$

Here, we regard $u$ and $v$ as coefficients, and apply [10, Theorem 5.3] by (3.58). Then, we obtain

$$
|u|_{\Omega \times(T+\tau, T+1)}^{(2+\theta)} \leq\left|u \zeta_{T}\right|_{\Omega \times(T, T+1)}^{(2+\theta)} \leq C_{56}
$$


or

$$
|u|_{\Omega \times\left(\tau, T_{\max }\right)}^{(2+\theta)} \leq C_{57} .
$$

On the other hand we have the parabolic regularity locally in time, so that we obtain

$$
|u|_{Q_{T_{\max }}}^{(2+\theta)},|v|_{Q_{T_{\max }}^{(2+\theta)}}^{\left(2 C_{58}\right.}
$$

Finally, if $T_{\max }<\infty$, the solution $(u, v)$ is extended after $T_{\max }$, a contradiction. Hence it holds that $T_{\max }=\infty$. We have the uniformly bounded solution globally in time, and the proof of Theorem 1.1 is complete.

\section{References}

[1] N. D. Alikakos, $L^{p}$ bounds of solutions of reaction-diffusion equations, Communications in Partial Differential Equations 4 (1979), no. 8, 827-868.

[2] P. Biler, Local and global solvability of some parabolic systems modelling chemotaxis, Advances in Mathematical Sciences and Applications 8 (1998), no. 2, 715-743.

[3] _ Global solutions to some parabolic-elliptic systems of chemotaxis, Advances in Mathematical Sciences and Applications 9 (1999), no. 1, 347-359.

[4] S. Childress and J. K. Percus, Nonlinear aspects of chemotaxis, Mathematical Biosciences. An International Journal 56 (1981), no. 3-4, 217-237.

[5] H. Gajewski and K. Zacharias, Global behaviour of a reaction-diffusion system modelling chemotaxis, Mathematische Nachrichten 195 (1998), 77-114.

[6] M. Giga, Y. Giga, and H. Sohr, L estimate for abstract linear parabolic equations, Japan Academy. Proceedings. Series A. Mathematical Sciences 67 (1991), no. 6, 197-202.

[7] D. Henry, Geometric Theory of Semilinear Parabolic Equations, Lecture Notes in Mathematics, vol. 840, Springer, Berlin, 1981.

[8] M. A. Herrero and J. J. L. Velázquez, A blow-up mechanism for a chemotaxis model, Annali della Scuola Normale Superiore di Pisa. Classe di Scienze. Serie IV 24 (1997), no. 4, 633-683 (1998).

[9] E. F. Keller and L. A. Segel, Initiation of slime mold aggregation viewed as an instability, Journal of Theoretical Biology 26 (1970), 399-415.

[10] O. A. Ladyženskaja, V. A. Solonnikov, and N. N. Uralt'seva, Linear and quasi-linear equations of parabolic type, Izdat. "Nauka", Moscow, 1968, English translation; American Mathematical Society, Rhode Island, 1968.

[11] T. Nagai, Blow-up of radially symmetric solutions to a chemotaxis system, Advances in Mathematical Sciences and Applications 5 (1995), no. 2, 581-601.

[12] _ Blowup of nonradial solutions to parabolic-elliptic systems modeling chemotaxis in twodimensional domains, Journal of Inequalities and Applications 6 (2001), no. 1, 37-55.

[13] T. Nagai and T. Senba, Global existence and blow-up of radial solutions to a parabolic-elliptic system of chemotaxis, Advances in Mathematical Sciences and Applications 8 (1998), no. 1, 145156.

[14] T. Nagai, T. Senba, and K. Yoshida, Application of the Trudinger-Moser inequality to a parabolic system of chemotaxis, Fako de l'Funkcialaj Ekvacioj Japana Matematika Societo. Funkcialaj Ekvacioj. Serio Internacia 40 (1997), no. 3, 411-433.

[15] V. Nanjundiah, Chemotaxis, signal relaying, and aggregation morphology, Journal of Theoretical Biology 42 (1973), 63-105.

[16] T. Senba, Blow-up of radially symmetric solutions to some systems of partial differential equations modelling chemotaxis, Advances in Mathematical Sciences and Applications 7 (1997), no. 1, 79 92. 
[17] T. Senba and T. Suzuki, Local and norm behavior of blowup solutions to a parabolic system of chemotaxis, Journal of the Korean Mathematical Society 37 (2000), no. 6, 929-941.

[18] Chemotactic collapse in a parabolic-elliptic system of mathematical biology, Advances in Differential Equations 6 (2001), no. 1, 21-50.

[19] _ Applied Analysis, Imperial College Press, London, 2004.

[20] T. Suzuki, Free Energy and Self-Interacting Particles, Progress in Nonlinear Differential Equations and Their Applications, vol. 62, Birkhäuser Boston, Massachusetts, 2005.

[21] H. Tanabe, Functional Analytic Methods for Partial Differential Equations, Monographs and Textbooks in Pure and Applied Mathematics, vol. 204, Marcel Dekker, New York, 1997.

Takasi Senba: Department of Applied Mathematics, Faculty of Technology, Miyazaki University, 1-1 Gakuen Kibanadai Nishi, Miyazaki-shi 889-2192, Japan

E-mail address: t0f302u@cc.miyazaki-u.ac.jp

Takasi Suzuki: Department of Mathematical Science, Graduate School of Engineering Science, Osaka University, 1-3 Machikaneyama-machi, Toyonaka-shi 560-8531, Japan

E-mail address: suzuki@sigmath.es.osaka-u.ac.jp 


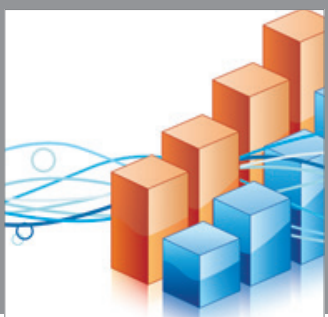

Advances in

Operations Research

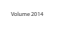

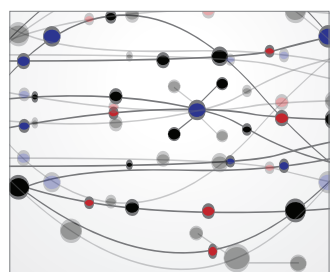

\section{The Scientific} World Journal
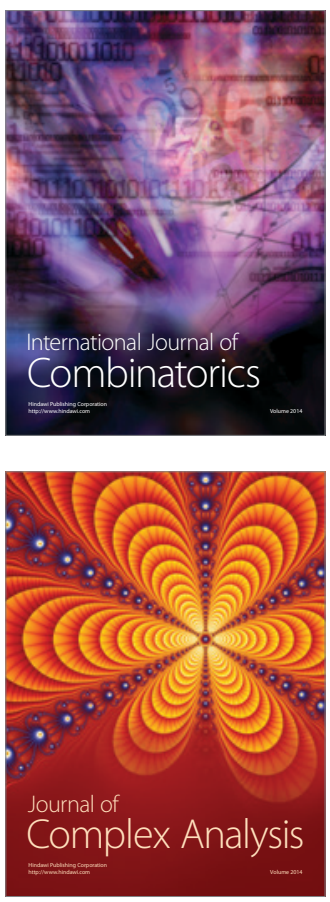

International Journal of

Mathematics and

Mathematical

Sciences
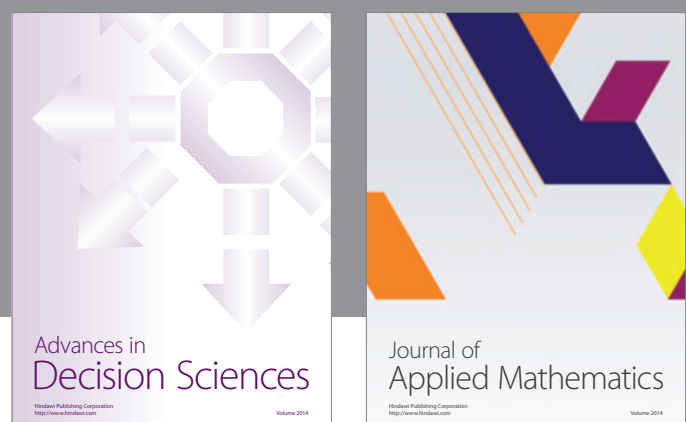

Journal of

Applied Mathematics
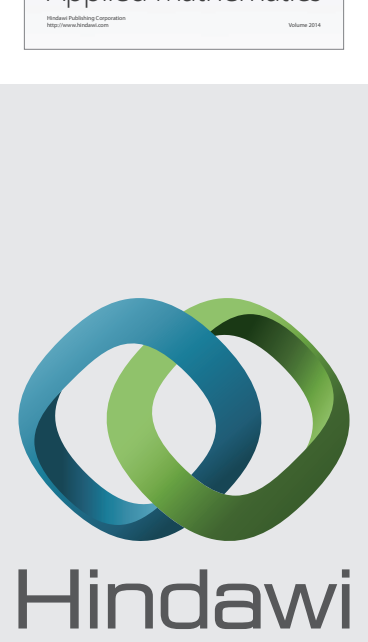

Submit your manuscripts at http://www.hindawi.com
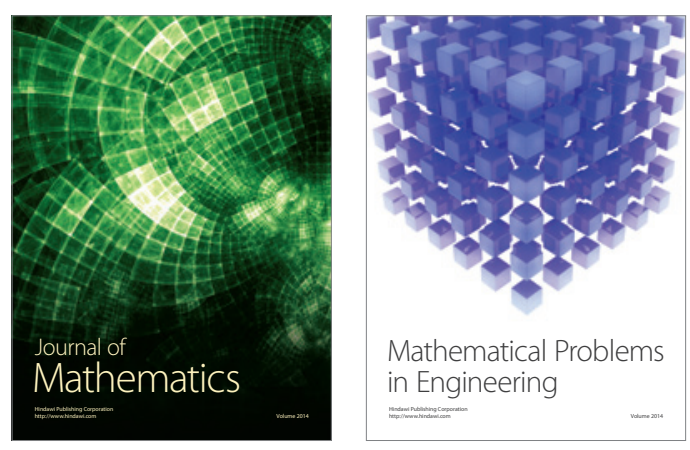

Mathematical Problems in Engineering
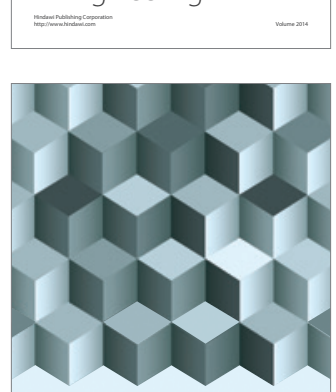

Journal of

Function Spaces
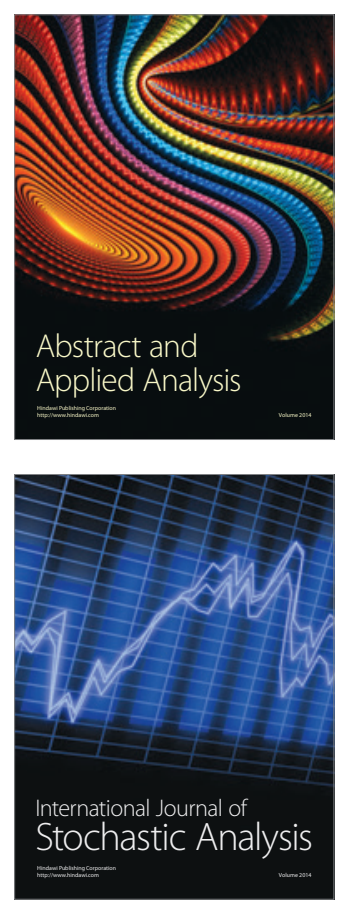

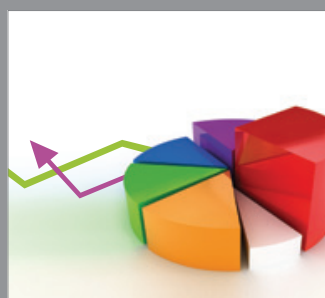

ournal of

Probability and Statistics

Promensencen
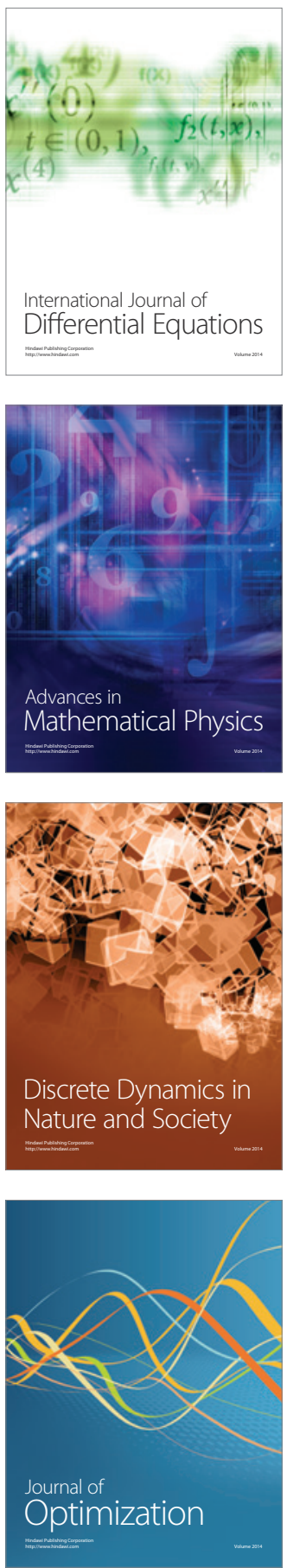